\title{
PHYLOGENOMIC RECONSTRUCTION SUPPORTS SUPERCONTINENT ORIGINS FOR LEISHMANIA
}

Kelly M. Harkins ${ }^{1,2 *}$, Rachel S. Schwartz ${ }^{3}$, Reed Cartwright ${ }^{3,4}$, and Anne C. Stone ${ }^{2,5}$

${ }^{1}$ Department of Anthropology

Human Paleogenomics Laboratory

University of California, Santa Cruz

Santa Cruz, CA, USA

${ }^{2}$ School of Human Evolution and Social Change

${ }^{3}$ The Biodesign Institute

${ }^{4}$ School of Life Sciences

${ }^{5}$ Center for Evolution and Medicine

Arizona State University

Tempe, AZ, USA

*Corresponding author: kmharkin@ucsc.edu 


\begin{abstract}
Leishmania, a genus of parasites transmitted to human hosts and mammalian/reptilian reservoirs by an insect vector, is the causative agent of the human disease complex leishmaniasis. The evolutionary relationships within the genus Leishmania and its origins are the source of ongoing debate, reflected in conflicting phylogenetic and biogeographic reconstructions. This study employs a recently described bioinformatics method, SISRS, to identify over 200,000 informative sites across the genome from newly sequenced and publicly available Leishmania data. This dataset is used to reconstruct the evolutionary relationships of this genus. Additionally, we constructed a large multi-gene dataset; we used this dataset to reconstruct the phylogeny and estimate divergence dates for species. We conclude that the genus Leishmania evolved at least 90-100 million years ago. Our results support the hypothesis that Leishmania clades separated prior to, and during, the breakup of Gondwana. Additionally, we confirm that reptile-infecting Leishmania are derived from mammalian forms, and that the species that infect porcupines and sloths form a clade long separated from other species. We also firmly place the guinea-pig infecting species, L. enrietti, the globally dispersed L. siamensis, and the newly identified Australia species from kangaroos as sibling species whose distribution arises from the ancient connection between Australia, Antarctica, and South America.
\end{abstract}

\title{
Keywords
}

Leishmania, phylogenomics, evolution 


\section{Introduction}

Leishmania is a genus of parasitic trypanosomatid protozoa responsible for the human disease complex, leishmaniasis, which is estimated to cause the ninth largest disease burden among infectious diseases. Leishmania spp. are transmitted primarily by sandflies to human hosts and animal reservoirs. Leishmaniasis is endemic to poverty-stricken countries, is rising in incidence by nearly two million cases annually, and lacks effective treatment or vaccine ${ }^{1}$. At least twenty Leishmania species transmitted via insect vector cause disease in humans with three main clinical manifestations: visceral, cutaneous and mucocutaneous. Despite extensive work since its first description in 1903, conflicting hypotheses persist regarding the evolution of Leishmania $^{2-6}$. This lack of consensus regarding current species relationships is due to incongruence between molecular and non-molecular data, as well as among molecular studies ${ }^{7,8}$. Additionally, available sequence data are skewed towards human-infecting species; comparatively scarce data from wild reservoir/host populations limits evolutionary reconstructions.

Thus, the majority of molecular phylogenetic studies of Leishmania have relied on a few genetic loci to infer evolutionary relationships ${ }^{15}$. These limited data may not accurately reflect the true history of the genus and also provide limited information with which to estimate the age of splits between clades ${ }^{9,16}$. The evolutionary history of this genus can be resolved with larger molecular datasets and by using these data to estimate the timing of the splits among clades within the phylogeny ${ }^{9-14}$. 


\section{Proposed origins hypotheses}

Leishmania are parasites that require two species for a complete life cycle: an insect vector and vertebrate host. Before the development of molecular biology techniques, the genus was divided into two subgenera based upon where the parasite developed within the vector (midgut vs. hindgut) ${ }^{17}$. These subgenera are Leishmania, consisting of all Old World species and one species complex found in the Americas, and subgenus Viannia, comprised exclusively of New World species. The species that infect Old World reptiles have since been placed in the subgenus Sauroleishmania ${ }^{18}$ while many other Leishmania species, including newly described lineages, remain unclassified.

The biogeography of vectors and distinct vertebrate hosts informs hypotheses for the origins of the genus. Three hypotheses have been proposed for the origin of Leishmania. The Palaearctic hypothesis assumes an origin in Cretaceous lizards with recent migrations to the Nearctic and Neotropics across land bridges. This hypothesis suggests that Sauroleishmania form a clade that is sister to all other species and is supported by non-molecular data, namely host-phylogenies, biogeography, and evidence of an ancestral parasite, Paleoleishmania, found fossilized in Cretaceous amber in modern-day Burma that is similar to species restricted to New World mammals $^{7,8,19-21}$.

Alternatively, Leishmania has been hypothesized to originate in the Neotropics. This hypothesis is supported by sequence-based phylogenies ${ }^{4,10-12,22}$. In these phylogenies, New World species are ancestral to those found in the Old World. Thus, the reptile-infecting forms are derived from mammalian forms of the parasite rather than being ancestral to them, contrary to the Palearctic 
hypothesis. The Neotropical hypothesis, however, requires two separate intercontinental migrations of the parasites: first the ancestor of Leishmania / Sauroleishmania to the Old World, followed by the migration of a member of the Leishmania subgenus back to the New World. Alternatively, there might have been two migrations into the Old World: once by an ancestor of Sauroleishmania and second by the ancestor of the Old World Leishmania species. The timing of these migrations must have coincided with appropriate land bridges.

Finally, the multiple origins hypothesis suggests division of Leishmania into two lineages on Gondwana ${ }^{9}$. One lineage led to the subgenera Leishmania, Viannia, and Sauroleishmania (termed Euleishmania), while the second diverged into all other species, which to date are restricted to New World mammals (Paraleishmania) ${ }^{4}$. The breakup of Gondwana subsequently separated the ancestors of the Viannia subgenus and the Leishmania and Sauroleishmania subgenera in South America and Africa, respectively. The amount of diversity observed between the Leishmania and Viannia subgenera has been cited as evidence for vicariance due to the separation of Africa from the Neotropics ${ }^{23}$. L. Leishmania spp. (e.g. L. Leishmania amazonensis) were subsequently brought from Africa through Eurasia to North America. Due to the insect vector's short lifecycle and weak flying ability this process must have occurred when conditions were conducive to vector survival, and the migration of vertebrate hosts ${ }^{24}$. The most recent date of an introduction of Leishmania subgenus into the Neartic via Beringia would have been the mid-Miocene when temperatures were warm enough for sandfly survival ${ }^{13}$.

This study represents the first phylogenomic analysis of Leishmania, employing over 200,000 variable sites and 49 genes from across the genome. This large dataset counters previous 
challenges suggesting that different substitution rates in different genes can confound estimates

of relationships among Leishmania ${ }^{9}$. The size of the dataset also allows us to estimate the timing of divergence among clades, which until now has been purely speculative. Our results support the hypothesis that Leishmania clades separated prior to, and during, the breakup of Gondwana. Additionally, we confirm that reptile-infecting Leishmania are derived from mammalian forms, and that the species that infect porcupines and sloths form a clade long separated from other species. We also firmly place the guinea-pig infecting species, L. enrietti, the globally dispersed L. siamensis, and the newly identified Australia species from kangaroos as sibling species whose distribution arises from the ancient connection between Australia, Antarctica, and South America.

\section{Materials and Methods}

\section{Whole genome shotgun sequencing}

We sequenced whole genomes of twelve species of Leishmania and one species of Endotrypanum (Table 1). Organisms were grown as promastigotes ${ }^{25}$ at $22^{\circ} \mathrm{C}$ in Schneider's Medium supplemented with $20 \%$ heat inactivated FCS and $17.5 \mathrm{mg} / \mathrm{mL}$ gentamycin. The cells were pelleted and washed twice in phosphate-buffered saline (PBS) and gDNA extracted according to established methods. The cells were incubated in lysis buffer (100mM NaCL, $10 \mathrm{mM}$ TrisCl $\mathrm{pH}$ 8.0, 0.5mM EDTA, 0.5\% SDS, 0.1mg/ml fresh Proteinase K) for 12-18hrs at 50C, and purified via phenol/chloroform/isopropyl alcohol. Extract was incubated in RNase A $(10 \mathrm{mg} / \mathrm{ml})$ for $2.5 \mathrm{hrs}$ at $37 \mathrm{C}$ and dialyzed overnight at $4 \mathrm{C}$ with 3 changes of PBS buffer. DNA concentration was evaluated with spectrophotometry (Beckman Coulter DU730) and re-extracted if contaminated with phenol. Paired-end reads (100 bp) were sequenced on an Illumina 
HiSeq2000 by the University of Arizona Genome Core. The number of pairs of reads for each species ranged from 2.1-14.3 million, or 10 - 67x average coverage for the $\sim 34$ million $b p$ genome.

Additional shotgun genomic data were downloaded from the European Nucleotide Archive (ENA; Table S1). These data represent all publicly available genome data per Leishmania taxon.

\section{Phylogenetically informative data}

Variable sites from across the genome

Although reference genomes are available for Leishmania, initial attempts to align shotgun sequencing reads to these references resulted in low alignment rates. Therefore, to extract phylogenetically informative data from the available shotgun sequences, we used the bioinformatics pipeline SISRS ${ }^{26}$. This method identifies sites that are fixed for each species and variable across species to construct a multiple-species alignment. SISRS determines the nucleotide each species has at a site via strict consensus. If this fails or there is no data for that site in a species, the information is considered "missing". We produced dataset VS-m6 allowing up to six taxa to have missing information per site (missing data were allowed to ensure sufficient data to determine the phylogeny). To ensure that linked sites would not bias our results, we subsampled this dataset by sampling only one site per sequence fragment, producing dataset VS-m6s. Finally, we produced an additional alignment, VS-t80, with no missing data allowed, but with a lower calling threshold of 0.8 , i.e. $80 \%$ of bases for that taxon must be one allele. 


\subsubsection{Additional gene data}

Because branch length estimates can be incorrect for variable site datasets, we developed an additional dataset of 49 genes with putative or known function. This dataset also allowed us to compare the results of two approaches to estimate the Leishmania phylogeny. We first obtained these genes from the L.V. braziliensis MHOM/BR/75/M2904 or M2903 reference genome (Table 2). The sequence of each gene for all other species was then extracted from the shotgun sequencing reads using the following pipeline: (1) Reads from each species were aligned to the reference genes using Bowtie ${ }^{27}$. (2) We used the mpileup feature of SAMTools ${ }^{28}$ to obtain the information from each read for each site. (3) The base for each site was identified based on whether at least $80 \%$ of the reads at that site contained a single base. (4) Alignments were adjusted using MAFFT ${ }^{29}$ with default settings. This pipeline is now automated as a part of SISRS.

We then added to the gene dataset all data available for two recently described Leishmania sp.: an isolate from Australian kangaroos (strain AM-2004) ${ }^{30}$ and L. siamensis ${ }^{31,32}$. These data were downloaded from GenBank. For AM-2004 we obtained partial sequences of three loci. For $L$. siamensis we obtained partial sequences of six loci, which includes two genes (Table S2).

\section{Phylogenetic analysis}

\section{Concatenated variable site analysis (3 datasets)}

The practice of concatenating thousands of likely unlinked sites into a single alignment ${ }^{33}$ can elide the variable genealogical histories of different chromosomal regions. However, variable sites cannot be partitioned by linkage to separate the history of genes from the history of the 
species, and it is computationally challenging to consider each site separately for a dataset of this size. We thus treated the SISRS variable site data as a single concatenated locus ${ }^{34}$. For each dataset, we constructed a phylogeny using maximum likelihood (ML) in RAxML 8.0.20 ${ }^{35}$ with a General Time Reversible (GTR) model and substitution rates following a discrete gamma distribution with four categories for 1000 bootstrap replicates and allowing for ascertainment bias correction using the Lewis model ${ }^{36}$.

\section{Gene data}

For coding genes, partitioning by codon position was found to provide a better fit than by gene alone ${ }^{37}$. The best way to partition the data was determined by the program partitionfinder with default settings ${ }^{38}$. Input partitions were separate codon positions for each gene. We employed the Bayesian Information Criterion for model selection to avoid overparameterization. The resulting 20 partitions were used to estimate the phylogeny in a ML framework; this analysis was implemented in RAxML 8.0.20 ${ }^{35}$ using a GTR model with gamma distributed rate heterogeneity across sites, for 100 bootstrap replicates. This analysis was repeated five times with random starting seeds to identify the ML tree and avoid local optima.

\section{Individual gene analysis}

ML trees for each gene were constructed identically in RAxML with 1000 bootstrap replicates. Although concatenated multi-gene datasets are found to be robust in phylogenetic inference, even without explicitly accounting for large variations in rates, lengths and GC content, systematic biases in the dataset can lead to high support of the wrong tree ${ }^{39}$. We follow Gadagkar, et al. ${ }^{39}$ recommendation to report the gene support frequency for each given partition. 
A majority rule (MR) consensus tree was generated in RAxML from the best scoring ML trees of individual gene trees for which we had all ingroup taxa. When necessary the outgroup Crithidia was pruned using Newick Utilites ${ }^{40}$.

\section{Estimating divergence time}

We used two approaches to estimate the timing of divergences among clades. Time estimates allow us to evaluate the plausibility of the proposed hypotheses for the origin of Leishmania. Due to poor fossil preservation of Leishmania, no secure fossil calibration dates within the genus exist. Thus, our first approach was to add two outgroup species, Trypanosoma cruzi and $T$. brucei, to the concatenated gene dataset. The divergence date for these species is believed to be 100 million years (my) ago based on the timing of the split between Africa and South America ${ }^{14,41}$. Using our known tree for Leishmania with these two additional species, divergence times were estimated using RelTime ${ }^{42}$.

Our second approach was to use a calibration date of 40 million years for the split between $L$. enriettii, L. siamensis and Leishmania sp. Ghana with the Australian isolate Leishmania sp. AM2004, which corresponds to the breaking of the connection between South America and Australia via Antarctica. In this analysis we only used the sequences available for AM-2004 to avoid any effect of missing data on branch lengths. For this reason, we were able to include additional Leishmania taxa for which these few loci were also publicly available (Table S3). We constructed dated phylogenies using BEAST v1.8.2 ${ }^{43}$ with two unlinked partitions, one for 18s/ITS/5.8s and the other for RNA polymerase II large subunit. We estimated substitution models in jmodeltest ${ }^{44}$, and given those results, implemented a TN93 substitution model with 
rates estimated from a gamma distribution with four rate categories and a relaxed lognormal molecular clock for 18,000,000 generations (high ESS values and convergence were observed in Tracer $1.5^{45}$ at this point). A normally distributed prior with a mean of 40 my was specified for the node leading to Australia kangaroo isolate, Leishmania sp. AM-2004.

\section{Results}

\section{Phylogenetic analyses}

Variable sites data

The number of variable sites identified for each dataset were 215,644 (VS-m6), 18,312 (VSm6s), and 2,790 (VS-t80). Based on an alignment with the reference genome for L. tarentolae, we determined that these sites are distributed across the Leishmania genome. GC content was over $70 \%$, which is higher than the GC content estimated previously of 50-60\% (Peacock et al. 2007). The ML phylogeny for all of these datasets supports the Leishmania and Viannia subgenera as monophyletic clades; Sauroleishmania is also monophyletic, although only two taxa were available for phylogenetic analysis, and sister to the clade comprising the other two subgenera (Figure 1). L. enrietti is supported as sister to all other Euleishmania. L. hertigi, L. deanei, and Endotrypanum (Paraleishmania), form a clade that is sister to all other Leishmania lineages (Euleishmania).

\section{Partitioned genes}

A total of 70,447 sites in 49 genes were concatenated in the alignment. The resulting ML tree is identical to that for the variable sites data, with the exception of the placement of $L$. enrietti as sister to the clade containing the subgenera Leishmania and Sauroleishmania, rather than as 
sister to Euleishmania (Figure 2). However, this placement was supported by less than 50\% of individual gene trees. Interestingly, when ML trees were constructed separately from each codon position the phylogeny was identical to that constructed for the variable site data, although the third codon position phylogeny had low support at several nodes. Leishmania AM-2004 and L. siamensis are most closely related to L. enrietti.

\section{Divergence time estimates}

Divergence dates were first estimated using the gene dataset for Leishmania and two species of Trypanosoma, and Reltime with a 100 my calibration point at the split between two outgroup species, T. cruzi and T. brucei (Figure 3). The estimated date for the split of L. donovani-L. major was 24.2 mya,

We were unable to obtain genome data for the sister genus to Leishmania, which is Leptomanas; thus, we approximate the origin of Leishmania based on the timing of the split of the genus into two clades, and the divergence of Leishmania and Crithidia. These dates lead to an approximate origin of 90mya.

Secondly, divergence dates were estimated using available loci for the Australian species in BEAST v1.8.2 $2^{43}$ and setting a normal prior distribution of 40 my on the node ancestral to this isolate and other members of the "L. enriettii complex". In this case we estimated the median date of the split of $L$. donovani-L. major to be 21.2 (15.2-47.3 95\% HPD) mya. These results suggest an older origin of the genus between 140 and 119 mya (i.e. between the split between 
Leptomonas and Leishmania, and the split between Paraleishmania and Euleishmania) (Figure 4 and Figure S1).

\section{Discussion}

\section{Data}

Shotgun sequencing data of thirteen Leishmania isolates (this study) and eleven species available publicly enabled us to mine whole genome datasets for hundreds of thousands of phylogenetically informative sites. Previous molecular datasets used for phylogenetic reconstruction ranged from $1-31$ genes ${ }^{47}$. Rather than relying on a few known genes, these data were sampled across the genome. The SISRS method is particularly valuable for Leishmania sp., which like many other non-model organisms, do not have a suitable reference genome for this purpose.

\section{Phylogeny}

Our results place species of Old and New World subgenus Leishmania in a monophyletic clade separate from species of the exclusively New World subgenus Viannia. These results are consistent with other molecular-based trees, including those that place Sauroleishmania as sister to the Leishmania subgenus ${ }^{12,48}$.

Our genome-wide, variable-site tree places the L. enrietti genome, a parasite of the New World guinea pig, sister to all Euleishmania. This result is consistent with some prior molecular phylogenies ${ }^{2,10,22}$; however, it differs from early hypotheses that suggest L. enriettii is a member 
of the New World L. Leishmania subgenus ${ }^{49,50}$ or later work suggesting it is sister to the entire genus 20,51 .

When all codon positions are considered, the concatenated gene dataset produces a conflicting topology whereby L. enrietti is not basal to all Euleishmania but only to the Sauroleishmania / Leishmania subgenera. This conflict is not necessarily surprising given the short branches leading to the splits of L. enrietti and the Euleishmania clades, which often reflects incomplete lineage sorting and would lead to different relationships estimated from different genes. Adding more loci, as we have done with the variable site dataset, is advocated in this case to increase resolution ${ }^{52}$. It is interesting to note that strong support for the tree produced from the multigene data is obtained when using only the first and second codon position of the gene data, and lower support for this same topology results from separate analysis of third positions. Substitutions are expected to be most rapid at the third position ${ }^{53}$. At these time scales, saturation in the third position may negatively affect the phylogenetic signal, as reflected in the decrease of support.

As with nearly all other analyses to date, the molecular evidence does not support the taxonomic classification of Endotrypanum as a separate genus. Relative to molecular markers, there are few morphological characters used to classify unicellular organisms, calling their utility into question, when compared to molecular data ${ }^{54}$. Sequence data support their inclusion in the Leishmania genus, unless all other Paraleishmania species are misclassified. 


\section{Dates}

The calibration of 40 mya for the split between the "L. enriettii complex" and the Australian isolate Leishmania sp. AM-2004, like other fossil dates, provides only a minimum time since two species diverged. However, an examination of the resulting dates on other nodes within the tree, namely the split to $L$. donovani and L. major, is consistent with previously published dates. The dates for the split of $L$. donovani and L. major calculated using both datasets $(24.2$ and 21.1 mya respectively), with different calibration points, compare favorably to those published

previously (24.6-14.7 my) ${ }^{14}$. This result suggests that results at other nodes may be considered reasonably valid when evaluating hypotheses for the origin of Leishmania.

\section{Origins hypotheses}

\section{The Paleoarctic hypothesis}

Our results reject the Palaearctic origin hypothesis for Leishmania ${ }^{7,20}$. First, Sauroleishmania are not sister to all other Leishmania, as would be suggested by this hypothesis. Second, our estimated dates conflict with a Pliocene introduction of the subgenus Viannia to the Neotropics at $\sim 5$ mya after the reformation of the Panama isthmus. If Kerr ${ }^{7}$ is correct in asserting that reptiles were the first hosts of Leishmania in the Cretaceous, extinction events associated with the K-T boundary could have erased evidence of those lineages; however, this speculation does not affect our interpretation.

\section{The Neotropical hypothesis}

Our results also allow us to reject the Neotropical Origins hypothesis. Under this hypothesis, the current global distribution of Leishmania is a purely a result of dispersal through vector / 
reservoir migration and not vicariance. Lukes et al.'s (2007) version of the hypothesis proposes the ancestor of Paraleishmania evolved in the Neotropics between 46-36 mya and dispersed north. Similarly, Noyes et al. (1997) and Noyes (1998) propose that the Leishmania / Endotrypanum clade evolved in the Neotropics between 65-40 mya and dispersed to the Nearctic and to the Palearctic through land bridges; the Neotropics however were isolated at this time. Our results suggest an origin of the clade at least 90 mya, which is not consistent with these proposed dates.

\section{The Multiple Origins hypothesis}

The Multiple Origins scenario refers to the separation and subsequent independent evolution of the Viannia and Leishmania subgenera before 90-100 mya. This is the only formalized hypothesis that includes vicariance as a mechanism for the evolution of the major Leishmania clades. However, the authors are unclear about the emergence of Paraleishmania; while this group is clearly shown as diverging from Euleishmania prior to the separation of Viannia and Leishmania subgenera, they state that Paraleishmania migrated to the New World with the introduction of hystricomorph rodents (e.g. porcupines) in the "early Cenozoic". This explanation is unlikely because hystricomorph rodents do not appear in the fossil record until 23 mya (Paleobiology database, fossilworks.org).

We propose a modification and expansion of the multiple origins hypothesis, which we now term the Supercontinent hypothesis, for the origin of the Leishmania genus. In this scenario, the ancestor of Leishmania emerged from monoxeneous parasites (those found in a single host) on Gondwana ${ }^{51}$. As with the Multiple Origins hypothesis, the ancestors of Paraleishmania, and the 
Viannia and Leishmania subgenera emerged before separation of Gondwana $90-100$ mya and possibly already sustained a global distribution, as suggested by the placement and divergence of strains found in Asia and Africa, here L. siamensis, L. martiniquensis, and Leishmania sp. Ghana. The Supercontinent proposal is in agreement with speculations proposed by Shaw ${ }^{55}$ who suggested that an adaptation to mammals occurred around 90 mya when mammals began to radiate and Africa became fully isolated. Additionally, levels of genetic diversity between the Viannia subgenera and Sauroleishmania / Leishmania subgenera have been cited previously as a reflection of vicariance after the separation of South America and Africa ${ }^{23}$. These speculations are consistent with the estimated divergence dates presented here using two methods with two types of datasets, one genome-wide and one gene-based, with separate calibration information, respectively.

Only one migration of a lineage in the Leishmania subgenus back to the New World is required by this hypothesis (notwithstanding the historical transfer of L. infantum to the New World by European settlers, often termed L. infantum chagasi ${ }^{56,57}$ ). The global distribution of the phlebotomine sandfly genera that almost exclusively serve as vectors for the parasite likely resulted from the breakup of Pangaea and subsequent continental splintering ${ }^{58,59}$.

Our results are consistent with Early Cretaceous fossils of Paleoleismania proterus found in sandflies trapped within Burmese amber $\sim 100$ mya, which are reportedly evidence of the first digenetic trypanosomatids ${ }^{21}$. There is no way to confirm the genus but the organisms are morphologically similar to Leptomonas, the sister of Leishmania. Interestingly, this species is believed to be associated with reptile hosts. 
The recent discovery of $L$. siamensis and other linages that fall within the "L. enriettii complex", a clade basal to all Euleishmania, in humans and other mammals in North America, Europe, West Africa and Asia further highlights the plausibility of an ancient global dispersal predating continental splintering; because few data are currently available for all lineages within the new $L$. enriettii clade, further work is necessary to rule out recent introduction of Leishmania to those regions. However, the placement of AM-2004 as sister to lineages found in the New World ( $L$. enriettii) is consistent with the biogeography of other groups separated by the breakup of Australia, Antarctica and southern South America (e.g. the plant genus Nothofagus). The Supercontinent hypothesis for Leishmania genus also draws parallels with a related kinetoplastid parasite, Trypanosoma. Hamilton, et al. ${ }^{60}$ hypothesize a southern-supercontinent origins in which T. brucei evolved in Africa and T. cruzi in the New World following the breakup of Gondwana. Divergent lineages of Trypanosoma has also been found in Australia ${ }^{61}$, therefore much like the southern-supercontinent hypothesis for Trypanosoma evolution, the position of the kangaroo Leishmania isolate is critical in our origins scenario. This parallel pattern of evolution between related parasites Trypanosoma and Leishmania potentially suggests similar evolutionary processes.

The most ancestral lineages of Leishmania - those crucial to resolve the evolutionary history of the genus - are not only those typically found in wild reservoir populations, but also the samples for which fewest data exist. Animal reservoirs are critical for maintaining Leishmania in the wild. Sampling strategies refocusing on wild host and vector populations, recently urged by many researchers, will shed light on the deepest nodes of the phylogeny and ultimately, on the processes of zoonotic transfer to humans. Until additional NGS data are available from 
unclassified and newly described taxa, we offer genome wide data and multiple approaches to estimate divergence dates further contributes to our understanding of the evolution of Leishmania.

\section{Acknowledgements}

Genomic DNA from Leishmania isolates was obtained from the lab of Diane McMahon-Pratt at the Yale School of Public Health and from Lucille Floeter-Winter at the University of Sao Paolo. This work was supported by a National Science Foundation Doctoral Dissertation Improvement Grant [grant number BCS-1232582 to K. Harkins and A. Stone], School of Life Science (ASU) [R. Cartwright], and a National Science Foundation Advances in Bioinformatics Grant [grant number DBI-1356548 to R. Cartwright]. Sudhir Kumar and Jay Taylor provided guidance and feedback on the development of the project. 


\section{Figures}

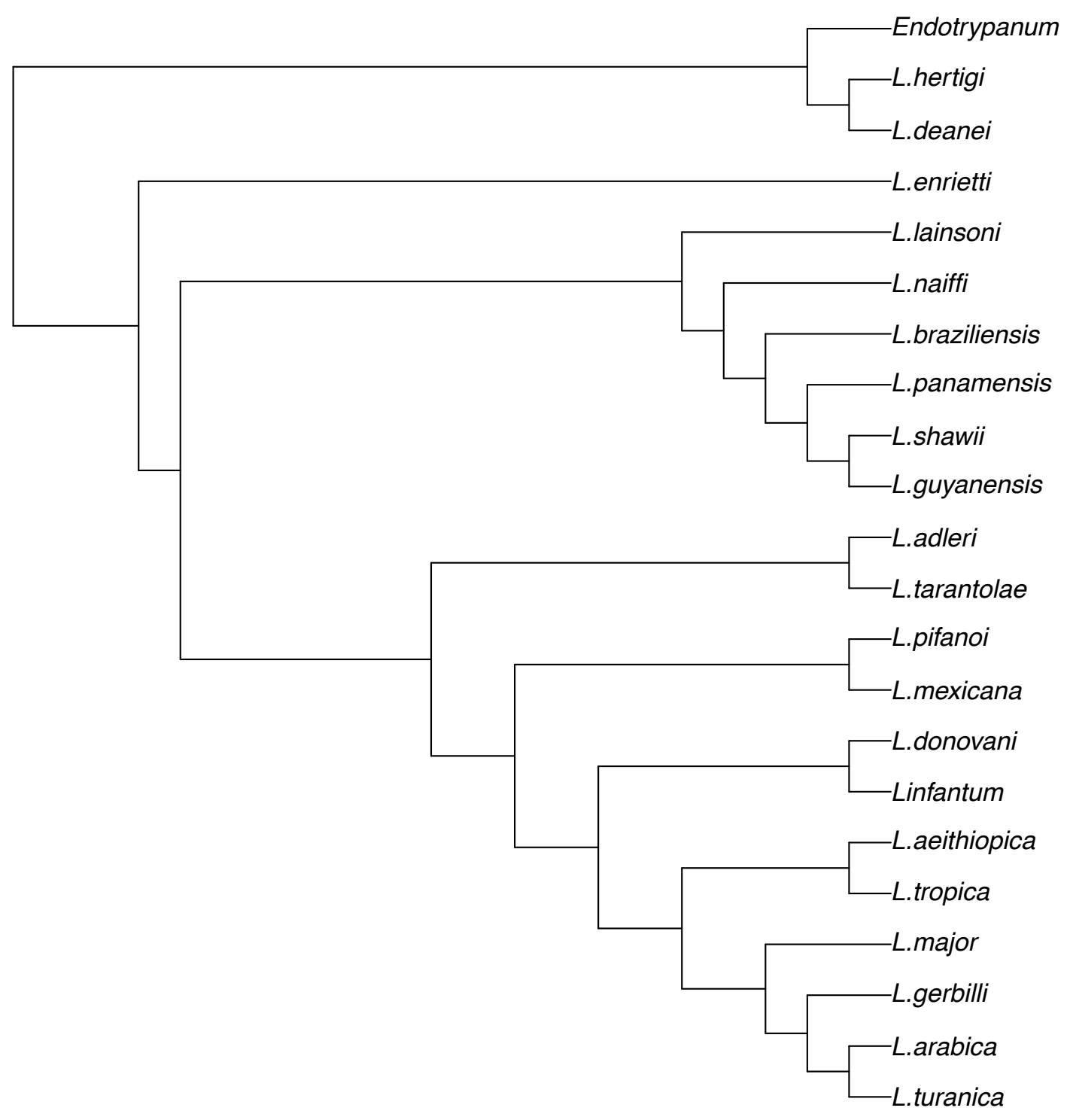

Figure 1: Maximum likelihood tree based on 215,644 variable sites from across the genome. Data were identified using SISRS ${ }^{26}$. Up to six samples were allowed to have data missing or be ambiguous for any given site. The phylogeny was constructed with RAxML 8.0.20 ${ }^{35}$ using the correction when only variable sites are present ${ }^{36}$. Bootstrap support values are 100 at every node. Identical results were obtained using a dataset containing only one site per sequence fragment, and one with no missing data allowed, but with a lower calling threshold of $80 \%$. 


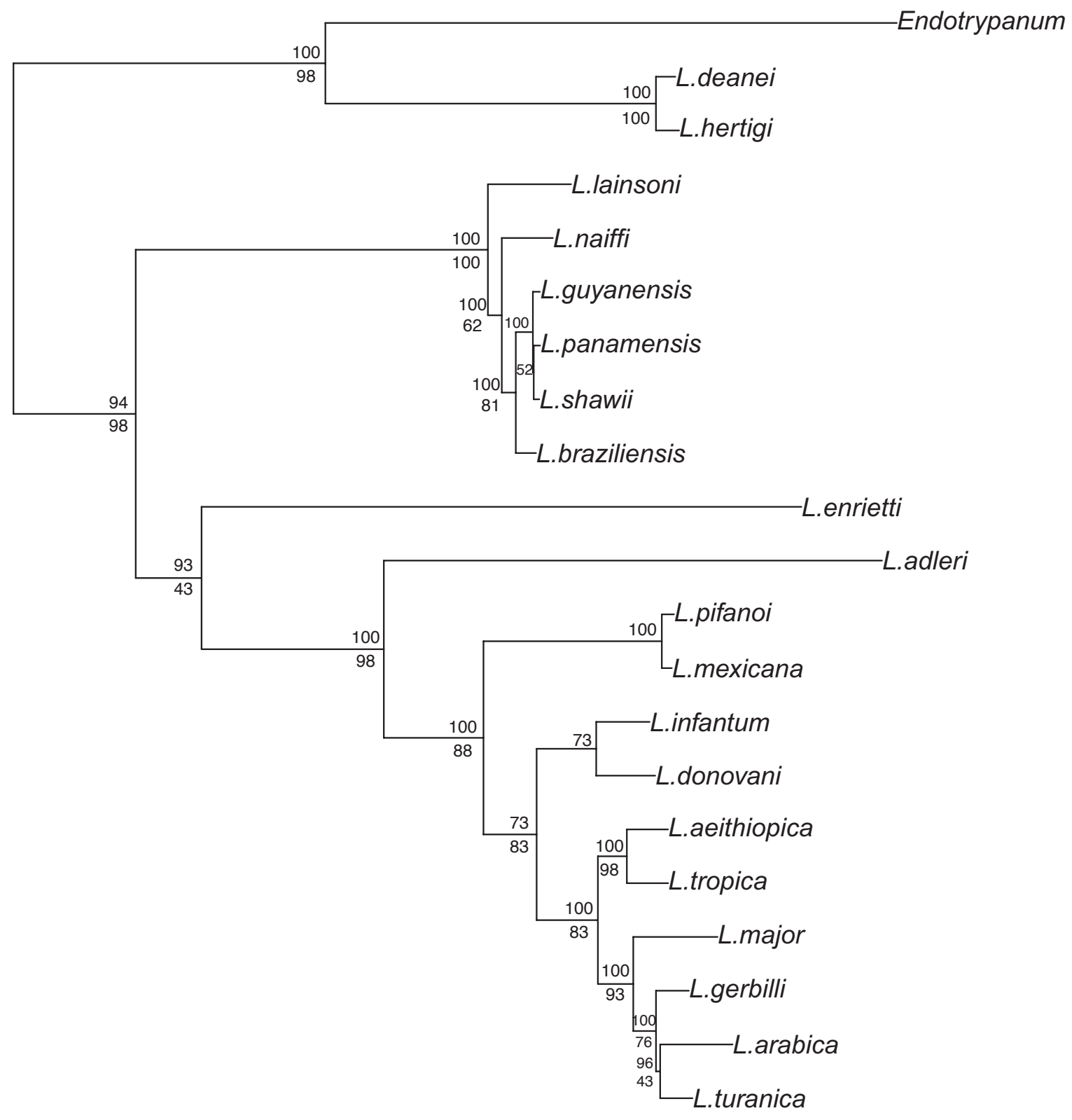

Figure 2: Maximum likelihood tree of Leishmania based on 49 genes. The phylogeny was constructed with RAxML 8.0.2035; data were partitioned by gene and codon position, with individual partitions grouped together using partitionfinder ${ }^{38}$. Top and bottom node labels indicate bootstrap support from 100 replicates and the percent of genes that support that node when the phylogeny for each gene was calculated individually, respectively. 


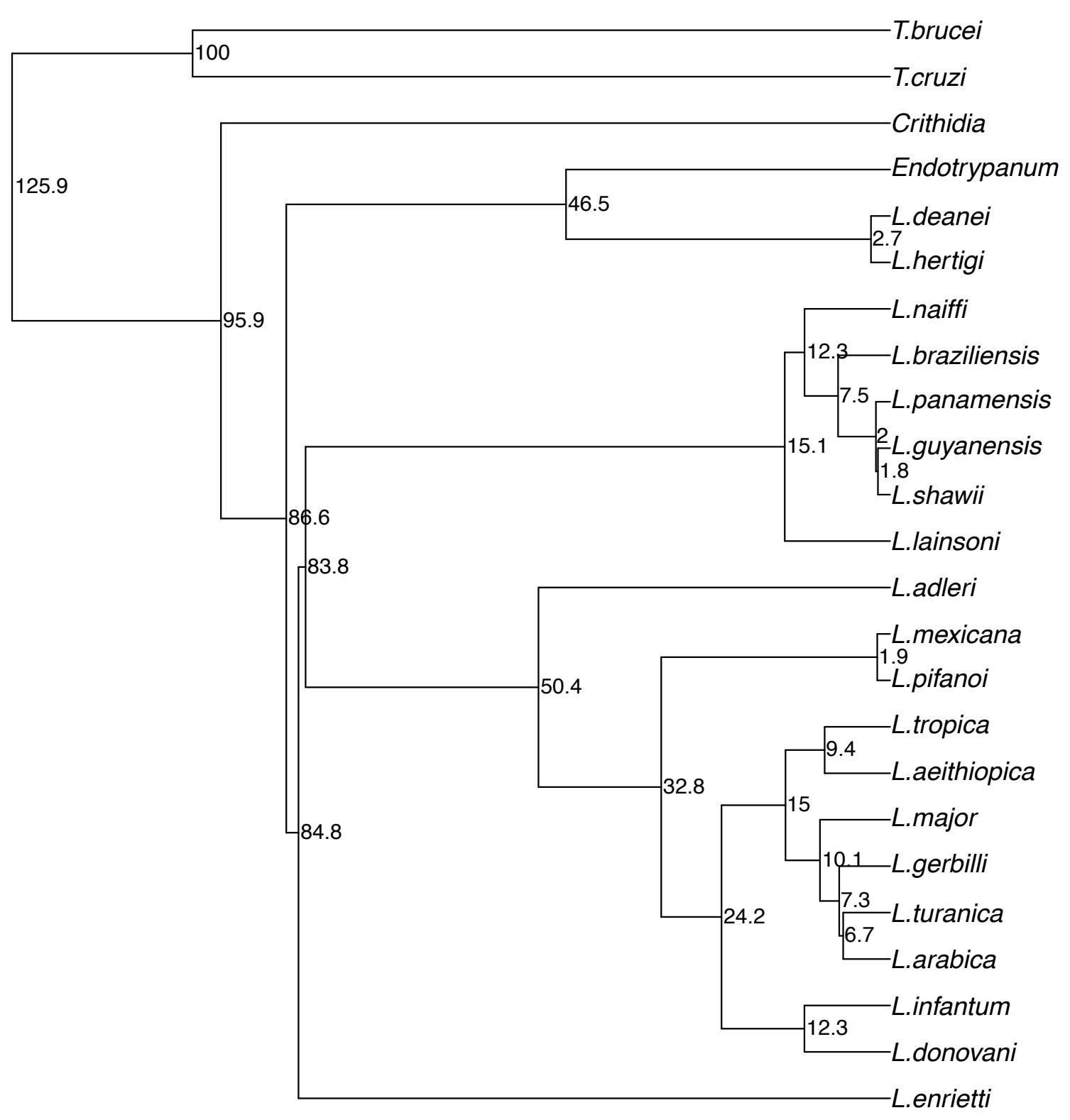

Figure 3: Dated phylogeny based on 49 genes. Constructed with Reltime using the calibration date of 100 mya for the split of Trypanosoma brucei and T. cruzi. Values at nodes are in million of years. 


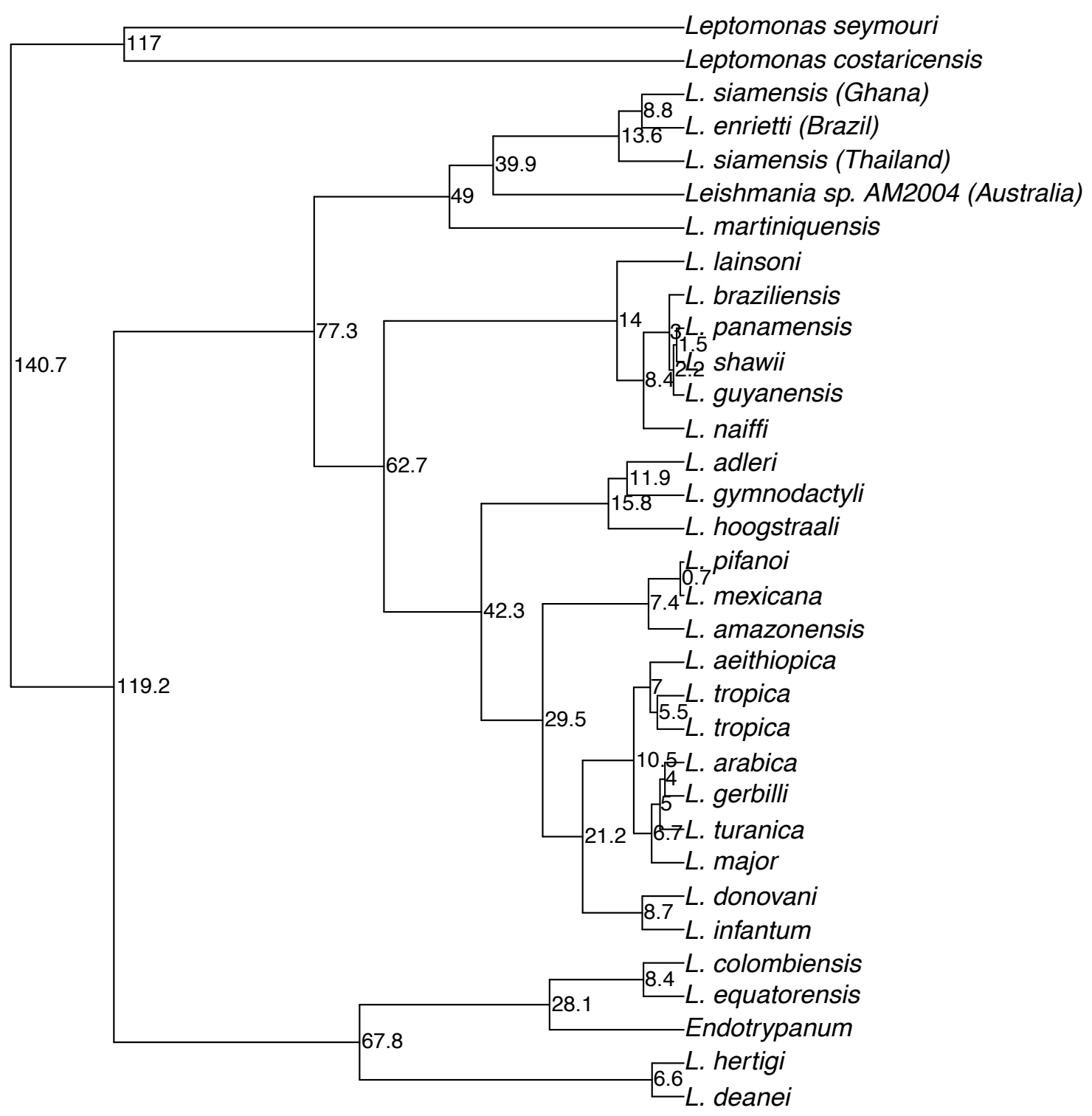

Figure 4: Phylogeny constructed using the loci available for L. $s p$. AM-2004 in BEAST with a relaxed molecular clock. Node values denote millions of years before present. See Figure S1 for posterior probability and 95\% HPD error bars. This phylogeny places the Australia sample as sister to L.siamensis and L. enrietti. Setting the split between the Australia sample and those taxa at 40 mya (a minimum time for the loss of connection between Australia and South America via Antarctica) allows us to estimate dates for the rest of the phylogeny. In particular, we estimate the median age of the split between Leishmania and Leptomonas, its sister genus, to be at 119 mya, and the split between the subgenera Sauroleishmania / Leishmania and Viannia at 62.7 mya. This result suggests that the Leishmania genus emerged prior to the breakup of Gondwana, and the latter split was caused by the separation of Africa from South America. 


\section{Supplementary Material}

Table S1: Raw genome data downloaded from public online databases.

Table S2: Accession information of genes acquired for Leishmania sp. AM-2004 and L. siamensis.

Table S3: Accession numbers for additional taxa included in BEAST dating analysis for RNA polymerase II large subunit (Rpol), 18s, Internal Transcribed Spacer 1 (ITS) and 5.8s. All others taxa derive from WGS data listed in Table 1 and $\mathrm{S} 1$.

Figure S1: BEAST tree with 95\% HPD error bars for estimated divergence dates. Scale in millions of years. Node values are posterior probabilities. 


\section{References}

1 Alvar, J. et al. Leishmaniasis worldwide and global estimates of its incidence. PLoS One 7, e35671, doi:10.1371/journal.pone.0035671 (2012).

2 Noyes, H. et al. A previously unclassified trypanosomatid responsible for human cutaneous lesions in Martinique (French West Indies) is the most divergent member of the genus Leishmania ss. Parasitology 124, 17-24 (2002).

3 Schönian, G., Mauricio, I. \& Cupolillo, E. Is it time to revise the nomenclature of Leishmania? Trends in parasitology 26, 466-469, doi:10.1016/j.pt.2010.06.013 (2010).

4 Cupolillo, E., Medina-Acosta, E., Noyes, H., Momen, H. \& Grimaldi, G., Jr. A revised classification for Leishmania and Endotrypanum. Parasitol Today 16, 142-144 (2000).

5 Lainson, R. The Neotropical Leishmania species: a brief historical review of their discovery, ecology and taxonomy. Rev Pan-Amaz Saude 1, 13-32 (2010).

6 Van der Auwera, G., Fraga, J., Montalvo, A. M. \& Dujardin, J.-C. Leishmania taxonomy up for promotion? Trends in parasitology 27, 49-50 (2011).

7 Kerr, S. F. Palaearctic origin of Leishmania. Memórias do Instituto Oswaldo Cruz 95, 75 80 (2000).

8 Lysenko, A. Distribution of leishmaniasis in the Old World. Bulletin of the World Health Organization 44, 515-520 (1971).

9 Momen, H. \& Cupolillo, E. Speculations on the origin and evolution of the genus Leishmania. Memórias do Instituto Oswaldo Cruz 95, 583-588 (2000).

10 Croan, D. G., Morrison, D. a. \& Ellis, J. T. Evolution of the genus Leishmania revealed by comparison of DNA and RNA polymerase gene sequences. Molecular and biochemical parasitology 89, 149-159 (1997).

11 Noyes, H. A., Morrison, D. A., Chance, M. L. \& Ellis, J. T. Evidence for a neotropical origin of Leishmania. Memórias do Instituto Oswaldo Cruz 95, 575-578 (2000).

12 Fraga, J., Montalvo, A. M., De Doncker, S., Dujardin, J.-C. \& Van der Auwera, G. Phylogeny of Leishmania species based on the heat-shock protein 70 gene. Infection, genetics and evolution : journal of molecular epidemiology and evolutionary genetics in infectious diseases 10, 238-245 (2010).

13 Stevens, J. \& Rambaut, A. Evolutionary rate differences in trypanosomes. Infect Genet Evol 1, 143-150 (2001).

14 Lukes, J. et al. Evolutionary and geographical history of the Leishmania donovani complex with a revision of current taxonomy. Proceedings of the National Academy of Sciences USA 104, 9375-9380 (2007).

15 Schönian, G., Cupolillo, E. \& Mauricio, I. in Drug Resistance in Leishmania Parasites (ed A. et al. Ponte-Sucre) 15-40 (Springer-Verlag, 2013).

16 Castresana, J. Topological variation in single-gene phylogenetic trees. Genome biology $\mathbf{8}$, 216, doi:10.1186/gb-2007-8-6-216 (2007).

17 Lainson, R. \& Shaw, J. J. Leishmaniasis of the New World: taxonomic problems. British medical bulletin 28, 44-48 (1972).

18 Saf'janova, V. The problem of taxonomy with Leishmania. Ser Protozool Sov Acad Sci Leningr 7, 5-109 (1982).

19 Kerr, S. F., Merkelz, R. \& Mackinnon, C. Further support for a Palaearctic origin of Leishmania. Memórias do Instituto Oswaldo Cruz 95, 579-581 (2000). 
20 Kerr, S. F. Molecular trees of trypanosomes incongruent with fossil records of hosts. Memórias do Instituto Oswaldo Cruz 101, 25-30 (2006).

21 Poinar Jr, G. Early Cretaceous trypanosomatids associated with fossil sand fly larvae in Burmese amber. Memórias do Instituto Oswaldo Cruz 102, 635-637 (2007).

22 Stevens, J. R., Noyes, H. A., Schofield, C. J. \& Gibson, W. The molecular evolution of Trypanosomatidae. Advances in parasitology 48, 1-56 (2001).

23 Fernandes, A. P., Nelson, K. \& Beverley, S. M. Evolution of nuclear ribosomal RNAs in kinetoplastid protozoa: perspectives on the age and origins of parasitism. Proc Natl Acad Sci U S A 90, 11608-11612 (1993).

24 Tuon, F. F., Amato Neto, V. \& Sabbaga Amato, V. Leishmania : origin, evolution and future since the Precambrian. FEMS Immunology \& Medical Microbiology 54, 158-166 (2008).

25 Wirth, D. F. \& Pratt, D. M. Rapid identification of Leishmania species by specific hybridization of kinetoplast DNA in cutaneous lesions. Proc Natl Acad Sci U S A 79, 6999-7003 (1982).

26 Schwartz, R. S., Harkins, K. M., Stone, A. C. \& Cartwright, R. A. A composite genome approach to identify phylogenetically informative data from next-generation sequencing. BMC bioinformatics 16, 193, doi:10.1186/s12859-015-0632-y (2015).

27 Langmead, B. \& Salzberg, S. L. Fast gapped-read alignment with Bowtie 2. Nat Methods 9, 357-359, doi:10.1038/nmeth.1923 (2012).

28 Li, H. et al. The Sequence Alignment/Map format and SAMtools. Bioinformatics 25, 2078-2079, doi:10.1093/bioinformatics/btp352 (2009).

29 Katoh, K. \& Standley, D. M. MAFFT multiple sequence alignment software version 7: improvements in performance and usability. Mol Biol Evol 30, 772-780, doi:10.1093/molbev/mst010 (2013).

30 Rose, K. et al. in International Journal for Parasitology Vol. 34 655-664 (2004).

31 Leelayoova, S. et al. Multilocus characterization and phylogenetic analysis of Leishmania siamensis isolated from autochthonous visceral leishmaniasis cases, southern Thailand. BMC microbiology 13, 60, doi:10.1186/1471-2180-13-60 (2013).

32 Kanjanopas, K. et al. Sergentomyia (Neophlebotomus) gemmea, a potential vector of Leishmania siamensis in southern Thailand. BMC infectious diseases 13, 333, doi:10.1186/1471-2334-13-333 (2013).

33 Philippe, H. et al. Resolving difficult phylogenetic questions: why more sequences are not enough. PLoS Biol 9, e1000602, doi:10.1371/journal.pbio.1000602 (2011).

34 Yoder, J. B. et al. Phylogenetic signal variation in the genomes of Medicago (Fabaceae). Sys Bio, Early View (2013).

35 Stamatakis, A. RAxML version 8: a tool for phylogenetic analysis and post-analysis of large phylogenies. Bioinformatics 30, 1312-1313, doi:10.1093/bioinformatics/btu033 (2014).

36 Lewis, P. O. A likelihood approach to estimating phylogeny from discrete morphological character data. Syst Biol 50, 913-925 (2001).

37 Mueller, R. L., Macey, J. R., Jaekel, M., Wake, D. B. \& Boore, J. L. Morphological homoplasy, life history evolution, and historical biogeography of plethodontid salamanders inferred from complete mitochondrial genomes. Proc Natl Acad Sci US A 101, 13820-13825, doi:10.1073/pnas.0405785101 (2004). 
38 Lanfear, R., Calcott, B., Ho, S. Y. \& Guindon, S. Partitionfinder: combined selection of partitioning schemes and substitution models for phylogenetic analyses. Mol Biol Evol 29, 1695-1701, doi:10.1093/molbev/mss020 (2012).

39 Gadagkar, S. R., Rosenberg, M. S. \& Kumar, S. Inferring species phylogenies from multiple genes: concatenated sequence tree versus consensus gene tree. Journal of experimental zoology. Part B, Molecular and developmental evolution 304, 64-74, doi:10.1002/jez.b.21026 (2005).

40 Junier, T. \& Zdobnov, E. M. The Newick utilities: high-throughput phylogenetic tree processing in the UNIX shell. Bioinformatics 26, 1669-1670, doi:10.1093/bioinformatics/btq243 (2010).

41 Stevens, J. R. \& Gibson, W. The molecular evolution of trypanosomes. Parasitology Today 15, 432-437 (1999).

42 Tamura, K. et al. Estimating divergence times in large molecular phylogenies. Proceedings of the National Academy of Sciences USA 109, 19333-19338 (2012).

43 Drummond, A. J., Suchard, M. A., Xie, D. \& Rambaut, A. Bayesian phylogenetics with BEAUti and the BEAST 1.7. Molecular Biology and Evolution 29, 1969-1973, doi:10.1093/molbev/mss075 (2012).

44 Darriba, D., Taboada, G. L., Doallo, R. \& Posada, D. jModelTest 2: more models, new heuristics and parallel computing. Nat Methods 9, 772, doi:10.1038/nmeth.2109 (2012).

45 Rambaut, A., Suchard, M. A., Xie, D. \& Drummond, A. J. Tracer v1.6. (2014).

46 Rambaut, A. FigTree: Tree Figure Drawing Tool Version 1.4. http://tree.bio.ed.ac.uk/software/figtree/ (2006).

47 Tschoeke, D. A. et al. The Comparative Genomics and Phylogenomics of Leishmania amazonensis Parasite. Evolutionary Bioinformatics Online 10, 131-153, doi:10.4137/EBO.S13759 (2014).

48 Dougall, A. M. et al. Evidence incriminating midges (Diptera: Ceratopogonidae) as potential vectors of Leishmania in Australia. International Journal for Parasitology 41, 571-579, doi:10.1016/j.ijpara.2010.12.008 (2011).

49 Lainson, R. On Leishmania enriettii and other enigmatic Leishmania species of the Neotropics. Mem Inst Oswaldo Cruz 92, 377-387 (1997).

50 Lainson, R. \& Shaw, J. J. in The leishmaniases in biology and medicine Vol. 1 Biology and Epidemiology (eds W Peters \& R Killick-Kendrick) 1-120 (Academic Press, 1987).

51 Yurchenko, V. Y., Lukes, J., Jirku, M., Zeledon, R. \& Maslov, D. A. Leptomonas costaricensis sp. n. (Kinetoplastea: Trypanosomatidae), a member of the novel phylogenetic group of insect trypanosomatids closely related to the genus Leishmania. Parasitology 133, 537-546, doi:10.1017/S0031182006000746 (2006).

52 Maddison, W. P. \& Knowles, L. L. Inferring phylogeny despite incomplete lineage sorting. Syst Biol 55, 21-30, doi:10.1080/10635150500354928 (2006).

53 Kimura, M. Evolutionary rate at the molecular level. Nature 217, 624-626 (1968).

54 Perkins, S. L., Martinsen, E. S. \& Falk, B. G. Do molecules matter more than morphology? Promises and pitfalls in parasites. Parasitology 138, 1664-1674, doi:10.1017/S0031182011000679 (2011).

55 Shaw, J. Ecological and evolutionary pressures on leishmanial parasites. Brazilian Journal of Genetics 20 (1997). 
56 Shaw, J. J. Further thoughts on the use of the name Leishmania (Leishmania) infantum chagasi for the aetiological agent of American visceral leishmaniasis. Memórias do Instituto Oswaldo Cruz 101, 577-579 (2006).

57 Marcili, A. et al. Phylogenetic relationships of Leishmania species based on trypanosomatid barcode (SSU rDNA) and gGAPDH genes: Taxonomic revision of Leishmania (L.) infantum chagasi in South America. Infect Genet Evol, doi:10.1016/j.meegid.2014.04.001 (2014).

58 Galati, E. Phylogenetic systematics of Phlebotominae (Diptera, Psychodidae) with emphasis on American groups. Bol Direc Malariol y San Amb 35, 133-142 (1995).

59 Filho, J. D. \& Brazil, R. P. Relationships of new world phlebotomine sand flies (Diptera: Psychodidae) based on fossil evidence. Mem Inst Oswaldo Cruz 98 Suppl 1, 145-149 (2003).

60 Hamilton, P. B., Teixeira, M. M. \& Stevens, J. R. The evolution of Trypanosoma cruzi: the 'bat seeding' hypothesis. Trends in parasitology 28, 136-141, doi:10.1016/j.pt.2012.01.006 (2012).

61 Stevens, J. R., Noyes, H. A., Dover, G. A. \& Gibson, W. C. The ancient and divergent origins of the human pathogenic trypanosomes, Trypanosoma brucei and T. cruzi. Parasitology 118 ( Pt 1), 107-116 (1999). 